\title{
Delivering Essential Surgical Care for Lower-limb Musculoskeletal disorders in the Low-Resource Setting
}

\author{
Deeptiman James $^{1,6}$ (i) Faye M. Evans ${ }^{2} \cdot$ Ekta Rai $^{3} \cdot$ Nobhojit Roy $^{4,5}$
}

Accepted: 10 June 2021 / Published online: 29 June 2021

(C) The Author(s) 2021

\begin{abstract}
Background Mismatched surgeon-anesthesiologist ratios often exist in low-resource settings making safe emergency essential surgical care challenging. This study is an audit of emergency essential procedures performed for lowerlimb (LL) musculoskeletal disorders (MSD) when an anesthesiologist was unavailable. It aims to identify strategies for safe anesthesia.

Methods A 5-year retrospective audit of emergency essential LL orthopedic procedures performed at remote mission hospital in Central India was performed. Out of necessity, a regional anesthesia (RA) protocol was developed in collaboration with anesthesiologists familiar with the setting. The incidence of intraoperative surgical and perioperative anesthesia complications when RA was administered by a surgeon was evaluated.

Results During this period, 766 emergency essential LL MSDs procedures were performed. An anesthesiologist was available for only 6/766. RA was administered by a surgeon for $283 / 766$. This included spinal anesthesia (SA) for 267/283 patients, peripheral nerve blocks for 16/283. Local infiltration and/or sedation was administered to 477/766. There were 17 intraoperative surgical complications. Anesthesia-related complications included 37/267 patients who required multiple attempts to localize subarachnoid space and SA failure in 9/267 patients all of whom had successful re-administration. Additional sedation and infiltration of local anesthetic was required in 5/267 patients.

Conclusion Remote pre-anesthesia consultation for high-risk patients, local surgeon-anesthesiologist networking, protocol-guided management, and dedicated short duration of training in anesthesia may be considered as an alternative for delivering RA for emergency essential surgery for LL MSDs due to unavailability of anesthesiologists.
\end{abstract}

Nobhojit Roy

nobhojit.roy@ki.se

Deeptiman James

deeptiman99james@gmail.com

Faye M. Evans

faye.evans@childrens.harvard.edu

Ekta Rai

drektarai@yahoo.com

1 Pediatric Orthopedic Unit, Department of Orthopedic, Christian Medical College Vellore, 1106, Paul Brand Building, Ida Scudder Road, Vellore 632004, India
2 Department of Anesthesiology, Critical Care and Pain Medicine, Boston Children's Hospital, Harvard Medical School, Boston, US

3 Department of Anesthesia, Head of Pediatric and ObGyn Anesthesia unit, Christian Medical College, Vellore, Tamil Nadu, India

4 WHO Collaborating Center for Research on Surgical Care Delivery in LMICs, BARC Hospital, HBNI University, Mumbai, India

5 Department of Global Public Health, Karolinska Institutet, Stockholm, Sweden

6 Christian Hospital Mungeli, Mungeli, Chhattisgarh 495334, India 


\section{Introduction}

Essential surgical care for musculoskeletal disorders listed in the Disease Control Priorities, $3^{\text {rd }}$ edition (DCP3) provides broad guidelines to define essential orthopedic care at first-level hospitals in low- and middle-income countries (LMICs) [1]. Realistically adherence to these guidelines while delivering essential surgical care is often challenging. One of the main issues is a lack of trained healthcare providers. This is especially true in India where despite efforts to try to improve healthcare, there are not enough healthcare professionals to meet the needs of the rural areas and disproportionately even fewer anesthesiologists. This deficiency can make providing safe surgical care challenging [2-5].

In India, there are reportedly only 1.27 anesthesiologists $/ 100,000$ population. This is far below the recommended 5/100,000 population recommended by the World Federations of Societies of Anesthesiologists (WFSA). This ratio is further skewed as the majority of anesthesiologists in India are reluctant to work outside urban areas $[5,6]$. Unlike many other areas of the world where physician and non-physician healthcare workers are trained to provide safe anesthesia and often work in the rural settings, in India only anesthesiologists are allowed to provide anesthesia care. Efforts are being made to increase the number and distribution of anesthesiologists in India; however, short-term solutions are needed to ensure safe anesthesia is available for emergency surgical care especially in the rural areas [7-9].

For many orthopedic procedures, regional anesthesia (RA) is a viable alternative to general anesthesia (GA) [10]. While not optimal, RA administered by non-anesthesiologist physicians (NAP) with focused training and standard proficiency is reportedly associated with a low risk of complications; however, safety and efficacy remain under-evaluated [11-13]. This study audits a single surgeon's experience providing RA and orthopedic care for emergency lower-limb musculoskeletal disorders (LL MSDs) in a low-resource setting (LRS), in the absence of an anesthesiologist.

\section{Material and methods}

A retrospective audit of surgical and anesthesia complications performed under RA for emergency surgery for LL MSDs was conducted after ethical approval from institutional board review (IRB Min No. 13279) by Christian Medical College, Vellore (8/26/2020). All procedures were performed at Christian Hospital, Mungeli, a small mission hospital located in rural central India from July 2011 to
June 2016, which is attached to Christian Medical College, Vellore. Procedures on patients less than 14 years, elective procedures, and those with anesthesia provided by an anesthesiologist were excluded.

During this period, this was the only hospital with a qualified practicing orthopedic surgeon for over 3000 square kilometers (catchment area $>700,000$ people). Ninety percent of the population reside in rural areas and over $70 \%$ of households belong to the lowest or second wealth quintiles [14-16]. Only one-tenth of villages are located within $10 \mathrm{~km}$ of a government-run first-level referral unit (FRU) and the nearest FRU for orthopedic trauma management is the tertiary referral center nearly $100 \mathrm{~km}$ away. Road connectivity in this rural district is predominantly via unsurfaced roads and dependent on erratic daytime public transport and limited ambulance service $[17,18]$.

A protocol for administration of RA by the surgeon for emergency LL MSDs when an anesthesiologist was not available, was developed by the surgeon (Table 1) in collaboration with 3 anesthesia colleagues. One had previously worked at the mission hospital while the other two had outreach experience. They all understood that there were times in which emergency surgeries were required and a local anesthesiologist would not be available. The surgeon had received 6 weeks of basic training in anesthesia including advanced airway techniques, administration of spinal anesthesia, and basic nerve blocks during his orthopedic residency. The remainder of his RA training was informal and included a mix of instructions (via phone, face-to-face) from anesthesia colleagues, videos, YouTube, and self-study (during his mission hospital tenure). There was no real-time consultation from local anesthesiologists available. As part of the protocol, patients who required emergency essential surgery for LL MSDs were given the option to have their surgery done at the local facility with a surgeon administered RA or referral to the regional center. Per the protocol, in high-risk cases a pre-operative consultation was obtained by the surgeon from an off-site anesthesiologist whether to offer RA or recommend transfer. High-risk cases were identified through a preanesthetic checklist (PAC). Patient monitoring was performed as per protocol (Table 1).

Conversion to GA was not feasible in the given circumstances. If SA wore off before completion of the surgical procedure or PNB was not sufficient, anesthesia was augmented with intravenous midazolam, ketamine, and/or local infiltration of lidocaine. This was directed by the surgeon but administered by the person responsible for monitoring the patient. Surgical intraoperative complications were defined as equipment malfunction or breakage, surgical device failure, lack of appropriate orthopedic 
Table 1 Protocol for NAP administration of regional anesthesia for emergency lower-limb orthopedic procedures in absence of an anesthesiologist

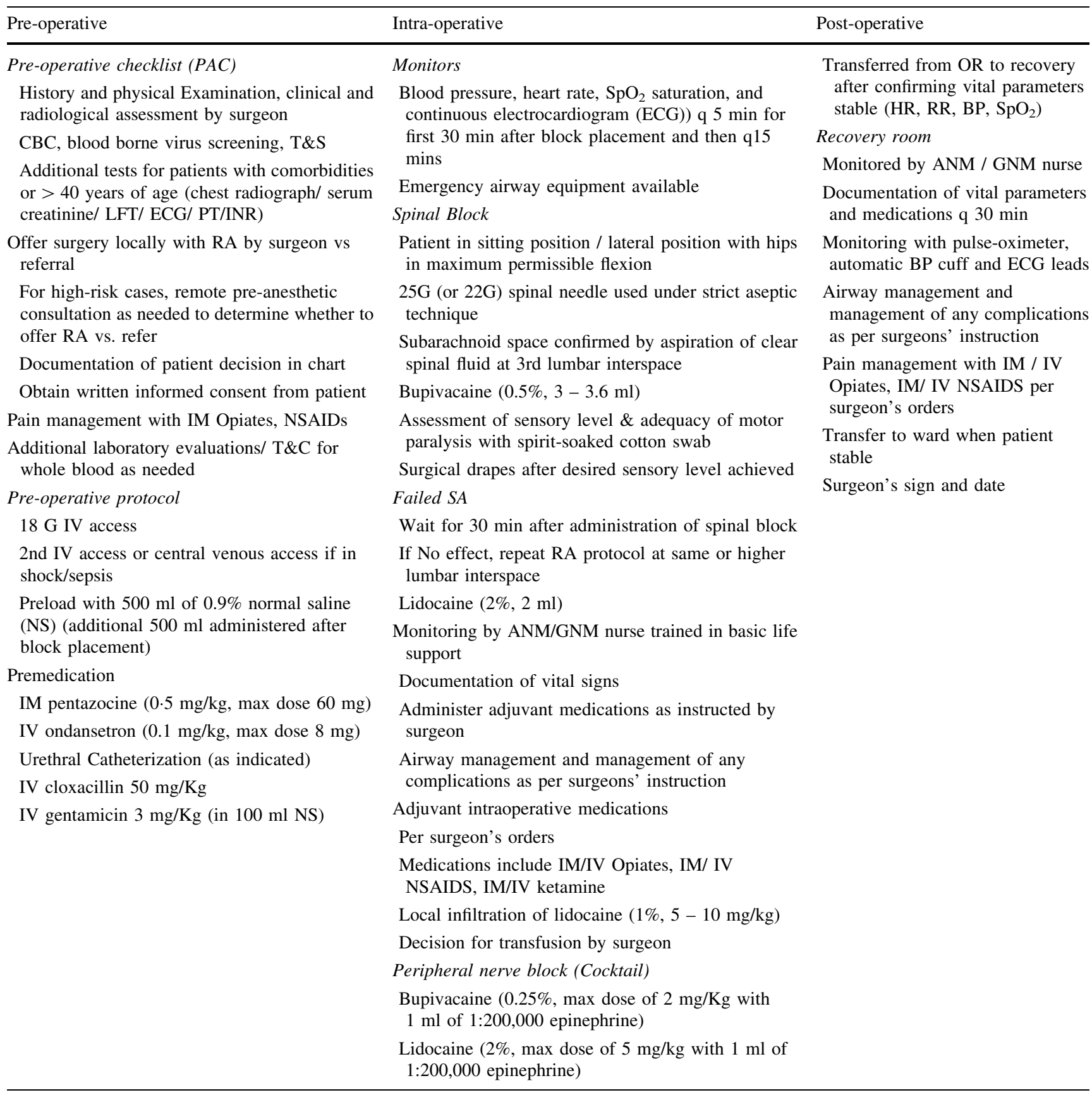

implant, unanticipated surgical events, technical difficulty, or errors leading to deviation from the surgical plan.

'Anesthesia-related complications' (ARC) were defined in relation to administration of RA and/or adequacy of the block for the surgical procedure. Complications related to administration included: difficulty with placement of RA (2 or more attempts), hypotension (systolic blood pressure $<90$ and/or diastolic blood pressure $<60$ ), bradycardia ( $\mathrm{HR}<50$ beats/minute), 'high spinal' with respiratory distress (requiring airway support); delay in recovery from RA, and persistent neurological deficits. The block was deemed inadequate if there was incomplete sensory or motor relaxation, tourniquet pain, or failed SA requiring additional anesthetic medications for amnesia/ analgesia. 
Table 2 Demographics of patients requiring regional anesthesia for emergency surgery for "essential" lower-limb musculoskeletal disorders $(N=283)$

\begin{tabular}{|c|c|}
\hline Median age (years; range) & $35(14-80)$ \\
\hline \multicolumn{2}{|l|}{ Gender } \\
\hline Male & $213(75.2 \%)$ \\
\hline Female & $70(24.8 \%)$ \\
\hline Median duration of surgery (minutes; IQR) & $120(120-180)$ \\
\hline \multicolumn{2}{|l|}{ Diagnosis } \\
\hline \multicolumn{2}{|l|}{ Trauma $(N=210)$} \\
\hline Open injury & $83(39.5 \%)$ \\
\hline Closed injury & $122(58.1 \%)$ \\
\hline Compartment syndrome & $3(1.4 \%)$ \\
\hline Morel Lavallee lesion & $2(0.1 \%)$ \\
\hline \multicolumn{2}{|l|}{ Musculoskeletal infection $(N=63)$} \\
\hline Septic arthritis & $15(23.8 \%)$ \\
\hline Acute osteomyelitis & $29(46 \%)$ \\
\hline Post-operative infections & $9(14.3 \%)$ \\
\hline Pyomyositis & $6(9.5 \%)$ \\
\hline Gas gangrene & $2(3.1 \%)$ \\
\hline Others & $2(3.2 \%)$ \\
\hline \multicolumn{2}{|l|}{ Tumors $(N=10)$} \\
\hline Malignant & $3(30 \%)$ \\
\hline Benign & $7(70 \%)$ \\
\hline \multicolumn{2}{|c|}{ Types of essential surgical procedures performed (DCP3) ${ }^{*}$} \\
\hline Fracture reduction & 103 \\
\hline Management of non-displaced fracture & 278 \\
\hline Irrigation and debridement of open fracture & 83 \\
\hline Placement of external fixator & 64 \\
\hline Fasciotomy & 3 \\
\hline Trauma related amputations & 3 \\
\hline Skin grafting & 26 \\
\hline Drainage of septic arthritis & 15 \\
\hline Debridement of osteomyelitis & 29 \\
\hline Wound debridement & 389 \\
\hline \multicolumn{2}{|l|}{ Other procedures (not included under DCP3) } \\
\hline Tumors & 10 \\
\hline Others & 18 \\
\hline \multicolumn{2}{|l|}{ Type of regional anesthesia } \\
\hline Spinal anesthesia & $267(94.3 \%)$ \\
\hline Sciatic PNB & $1(0.3 \%)$ \\
\hline Femoral PNB & $2(0.7 \%)$ \\
\hline Ankle PNB & $13(4.6 \%)$ \\
\hline
\end{tabular}

* Some cases required combination of multiple DCP3 procedures

A risk assessment was performed based on the numbers of ARCs that resulted in either adjuvant medications being administered and/or a change of surgical plan due to inadequate RA administered by the surgeon.

\section{Results}

Over the five-year audit period, 766 patients with LL MSDs underwent emergency essential surgery. Patient demographics and type of surgeries performed are reported in Table 2. An anesthesiologist was only available for 6 of these procedures which are not included in this audit. Two hundred and eighty-three procedures required RA which was performed by the surgeon. SA was administered in 267 (94.3\%) of these patients and sciatic, femoral, and ankle PNBs were administered in one, two, and 13 patients, respectively. The remaining 477 procedures were performed with ketamine and/or midazolam sedation administered by a nurse or medical doctor, and/or local infiltration by the surgeon. Intraoperative surgical complications were encountered in 17 patients (Table 3). ARCs were encountered in 46 patients who were administered SA and in one patient who underwent sciatic PNB. Difficulty with placement was encountered in 37 patients with an average of 2 attempts. SA failed in 9 patients and was successfully re-administered with $2 \%$ lidocaine. Transient post-spinal hypotension was encountered in 104 patients. In all cases, hypotension resolved with $0.9 \%$ normal saline boluses and rarely Mephentermine IV was needed. This was managed by the person monitoring the patient with

Table 3 Intraoperative complications

\begin{tabular}{ll}
\hline Regional anesthesia complications & \\
\hline Spinal anesthesia $(N=267)$ & $37(13.8 \%)$ \\
Difficulty in administering & $9(3.7 \%)$ \\
Failed anesthesia & $9(3.7 \%)$ \\
Re-administration & $5(1.8 \%)$ \\
Need for augmentation (ketamine/sedation) & $104(40 \%)$ \\
Hypotension & \\
Sciatic PNB $(N=1)$ & $1(100 \%)$ \\
Failed anesthesia & $1(100 \%)$ \\
Need for augmentation (ketamine/sedation) & \\
Femoral PNB $(N=2)$ & \\
No complications & $1(7.7 \%)$ \\
Ankle PNB $(N=13)$ & 17 \\
Need for augmentation (ketamine/sedation) & \\
Intraoperative surgical complications & 2 \\
Device failure & 4 \\
Image intensifier malfunction & 1 \\
Instrument/jig breakage & 3 \\
Fracture table attachment breakage & 5 \\
Implant not available & 2 \\
Technical difficulty and mal-reduction & \\
Implant pull-out requiring Re-operation & \\
\hline
\end{tabular}


direction by the surgeon. Since the transient hypotension quickly resolved with fluids and did not impact the surgical plan, it was not included as an ARC. No cardio-respiratory complications were noted (Table 3 ).

One patient with a closed femoral shaft fracture with severe mitral stenosis where SA was contraindicated was offered surgery under sciatic and femoral PNBs. However, anesthesia was inadequate for the patient to undergo intramedullary nailing. Hence, the surgical plan was revised to closed reduction with balanced traction [19].

$\mathrm{SA}$ required augmentation in 5 patients. In one of these patients, with ipsilateral femoral shaft and neck fractures, the surgical time exceeded $300 \mathrm{~min}$.[20] No anesthetic complications were encountered with femoral and ankle PNBs (Table 3).

\section{Discussion}

The prevalent practice of mandatory service obligation period after completion of postgraduate orthopedic training in India aims to address the skewed rural-urban distribution of orthopedic healthcare resources. For nearly a century, Christian Medical College Vellore has addressed this inequitable healthcare distribution by sending out providers to sustain and support healthcare in remote areas across the country. This commitment has ensured at least a minimal level of orthopedic surgical care in several remote areas $[2,3]$. Evolving socio-economic status and increased healthcare demands from the rural population, push for institutional accreditation, increased standards for obtaining Government health insurance coverage and more stringent supervision by regulatory bodies have led to an emphasis on quality-oriented, specialty driven approach without fully considering the realities and challenges of delivering safe surgical care in rural areas [1, 4-6]. This orthopedic clinic comprising of a physiotherapy and occupational therapy unit, a radiology suite with an X-ray machine, an Image Intensifier, the sole CT scan machine in the entire district, and operation theater equipped with only basic orthopedic equipment and sterilization facilities served more than 7 million people ( $>90 \%$ from rural areas).

Delivering safe and quality bellwether surgical care, in rural India is challenging. Recently trained orthopedic surgeons with limited experience, functioning as a singledoctor unit to provide care without an anesthesiologist is a routine narrative not just in India, but in many LMIC healthcare systems [6, 18, 21, 22]. Considering the projected shortfall and estimated time necessary to achieve the minimum goal of ensuring 20 Surgeons, Anesthesiologists and Obstetricians (SAOs)/100,000 population, rural areas will continue to have a shortage of physician anesthesiologists and will have to choose between declining care or providing emergency essential surgical care without an anesthesiologist or training a physician to give safe anesthesia without reaching full specialist status as they do in Australia and Canada [21, 23, 24]. The debate over non-physician anesthesia providers (NPA) empowerment remains controversial with skewed focus on difficult scenarios and poorer outcome but ignoring the fact that, not being able to provide emergency essential surgeries immediately to patients can result in worse outcomes for patients [13, 25-31].

Regional anesthesia is traditionally believed to be safer than GA. However, RA is not devoid of complications. To be performed safely it requires training, protocols must be followed and should be used in appropriate situations [11, 31-33]. Pawa et al. outlined the role of NAPs, and surgeons administered RA through additional training and guidelines, thus optimizing available resources to make safe anesthesia more accessible. Conversion to GA, advanced airway management, and managing unexpected complications are potential pitfalls [11]. Lewis et al. found inconclusive evidence to prove anesthesia delivered by NPAs was in any way inferior or more dangerous than when delivered by an anesthesiologist in over 1,500,000 patients in the USA [30]. Unlike the USA, most LMICs do not have specific guidelines or dedicated training for NPA; therefore, these results must be interpreted judiciously. Several studies have documented the relative safety of RA when administered by a NAP for emergency obstetric surgery where there is no anesthesiologist [29, 34, 35]. Lokossou et al., in a review of anesthesia services across 17 Sub-Saharan countries, have emphasized the training of NPAs to compensate for the critical lack of anesthesiologists [36]. Enright has encouraged increasing output of trainees from anesthesiology residency programs for rural posting as well as supervision and continued medical education for NPAs to overcome the deficit [37]. In contrast, Khan et al. have proposed a more equitable distribution of existing anesthesia workforce to ensure at least bellwether surgical procedures are carried out safely [38].

The present audit proposes NAP networking with offsite anesthesiologists for remote pre-anesthesia consultation in high-risk cases, development of NAP administered RA protocol and additional training is feasible. The role of a detailed pre-operative assessment, judicious patient selection, remote consultation and guidance from anesthesiologist colleagues, constructive team dynamics and reasonable skills to plan and execute surgical procedure promptly cannot be over-emphasized.

Surgical complications noted in this audit could be attributed either to the relative inexperience of the surgical team or limited resources. ARCs audited in this study are unique as they are determined from the surgeon's 
perspective in terms of ease of administration, adequacy of muscle relaxation, appropriateness of level, duration of action, and post-operative recovery. SA was administered in a sitting position as most patients could not be turned to the side due to pain. Hence, the need for multiple attempts for SA may have resulted from suboptimal positioning rather than inexperience. A SA failure of $3.7 \%$ observed in this study is comparable to the reported $4 \%$ failure rates among anesthesia trainees but higher than the reported $<$ $1 \%$ failure rates among experts [39, 40]. A detailed analysis of the cause of failure of SA is beyond the scope of this study. Hypotension is a common side effect of spinal anesthesia occurring in 16-33\% of cases [41]. While it was detected in $40 \%$ of our patients, it was expected and easily treated with fluids and from the surgeon's perspective did not result in any deviation from the perioperative protocol or surgical plan. For this reason, it was not included as an ARC. Lack of a real-time anesthesiologists' perspective of ARCs is an obvious limitation in this audit but then it would contradict the very need of this audit to assess the safety and efficacy of surgeon administered RA.

Though no conclusions can be drawn regarding the few PNBs audited in this study, it does show why clinical exposure and training of non-anesthesiologists in common PNB techniques should be explored [24, 34, 37]. The unique 'hands-on' training during an elective clinical posting in anesthesiology may have contributed to the successful administration of SA in this case and is a testament to our institution's commitment to training physicians for rural mission service.

This study highlights the concept of networking and remote pre-anesthesia consultation with off-site anesthesiologists to mitigate risk and provide safe anesthesia services in LRS. The rapid expansion of mobile network and familiarity with android based communication apps has made remote consultation with an off-site anesthesiologist feasible and cost-effective. However, this audit is inadequate to ascertain efficacy of remote pre-anesthesia consultations.

\section{Limitations}

This retrospective audit suffers from inherent selection bias, lack of standardized documentation, and a universal outcome grading system. Safety and efficacy of surgeon administered RA in this cohort cannot be compared with anesthesiologist administered RA and lack of an alternate benchmark for comparison poses a significant limitation in extrapolating our results to other low-resource settings. The results of this study cannot be generalized and must be interpreted based on local resources and limitations. In addition, the technical expertise of SA and PNB are highly variable and operator dependent. Effect of spinal needle gauge, patient positioning, demographic factors, and effect of the learning curve of the NAP on results were not assessed. Finally, this study is unable to quantify stress associated with delivering essential orthopedic surgical care without an attending anesthesiologist and its impact on surgical outcomes.

\section{Conclusion}

Regional anesthesia, especially SA proved to be an acceptable alternative for providing emergency and essential surgical care for LL MSDs in this low-resource setting, when there was no anesthesiologist. This solution must be explored further to solve the current crisis of anesthesiologist-deficit in LMICs. A qualified attending anesthesiologist cannot be replaced in a surgical team, and this study by no means implies or suggests that. Alternative measures including networking, 'remote pre-anesthetic consultation' and training of NAP in basic RA techniques in delivery of safe surgery need further evaluation.

Acknowledgements We acknowledge and thank Dr Rupa Anna, Consultant Anesthesiologist, Pune Adventist Hospital, Pune, India for her help and guidance in developing the pre-anesthesia checklist and protocol for regional anesthesia.

Funding Open access funding provided by Karolinska Institute. Nil.

\section{Declarations}

Conflict of interest The authors declare that they have no conflict of interest.

Open Access This article is licensed under a Creative Commons Attribution 4.0 International License, which permits use, sharing, adaptation, distribution and reproduction in any medium or format, as long as you give appropriate credit to the original author(s) and the source, provide a link to the Creative Commons licence, and indicate if changes were made. The images or other third party material in this article are included in the article's Creative Commons licence, unless indicated otherwise in a credit line to the material. If material is not included in the article's Creative Commons licence and your intended use is not permitted by statutory regulation or exceeds the permitted use, you will need to obtain permission directly from the copyright holder. To view a copy of this licence, visit http://creativecommons. org/licenses/by/4.0/.

\section{References}

1. Mock CN, Donkor P, Gawande A et al (2015) Essential surgery: key messages from disease control priorities. Lancet 385:2209-2219

2. Iyadurai R, Viggeswarpu S, Zachariah A (2019) Career destination and reason for career destination preferences among medical graduates from Christian Medical College vellore-does rural 
service obligation increase retention of medical graduates in rural service? J Fam Med Prim Care 8:2401

3. Mohan P, Kumar R (2019) Strengthening primary care in rural India: lessons from Indian and global evidence and experience. J Fam Med Prim Care 8:2169-2172

4. Weiser TG, Gawande A (2016) Excess surgical mortality: strategies for improving quality of care. In: Debas HT, Donkor P, Gawande A et al (eds) essential surgery: disease control priorities, third edition (volume 1). The International Bank for Reconstruction and Development / The World Bank, Washington

5. Divatia JV (2017) Safe anaesthesia for all Indians: a distant dream? Indian J Anaesth 61:531-533

6. Kempthorne P, Morriss WW, Mellin-Olsen J et al (2017) The WFSA global anesthesia workforce survey. Anesth Analg 125:981-990

7. Law TJ, Lipnick M, Joshi M et al (2019) The path to safe and accessible anaesthesia care. Indian J Anaesth 63:965-971

8. Mavalankar D, Sriram V (2009) Provision of anaesthesia services for emergency obstetric care through task shifting in South Asia. Reprod Health Matters 17:21-31

9. Mavalankar D, Callahan K, Sriram V et al (2009) Where there is no anesthetist-increasing capacity for emergency obstetric care in rural India: an evaluation of a pilot program to train general doctors. Int J Gynaecol Obstet 107:283-288

10. Capdevila X, Aveline C, Delaunay L et al (2020) Factors determining the choice of spinal versus general anesthesia in patients undergoing ambulatory surgery: results of a multicenter observational study. Adv Ther 37:527-540

11. Pawa A, El-Boghdadly K (2018) Regional anesthesia by nonanesthesiologists. Curr Opin Anaesthesiol 31:586-592

12. Wilson JM, Farley KX, Bradbury TL et al (2020) Is spinal anesthesia safer than general anesthesia for patients undergoing revision THA? analysis of the ACS-NSQIP database. Clin Orthop Relat Res 478:80

13. Committee O-L-L (2019) The lancet commission on global surgery - association of rural surgeons of India karad consensus statement on surgical system strengthening in rural India. Healthc (Amst) 7:7-9

14. Nandi S, Schneider H, Garg S (2018) Assessing geographical inequity in availability of hospital services under the state-funded universal health insurance scheme in Chhattisgarh state, India, using a composite vulnerability index. Glob Health Action $11: 1541220$

15. International Institute for Population Sciences (IIPS) (2010) District level household and facility survey (DLHS-3), 2007-08: India.Chhattisgarh: Mumbai: IIPS. http://rchiips.org/pdf/rch3/ report/ch.pdf. Accessed 8 Mar 2021

16. PROJECT IMPLEMENTATION PLAN 2012-13, CHHATTISGARH. http://cghealth.nic.in/health/programonitoring/PIPHealth Financing/PIP201213.pdf. Accessed 8 Mar 2021

17. Chhattisgarh road connectivity project: report and recommendation of the president to the board of directors. https://www.adb. org/sites/default/files/project-documents/52002/52002-001-rrpen.pdf. Accessed 8 Mar 2021

18. District disaster management plan-(DDMP) MUNGELI. http:// sdma.cg.gov.in/Mungeli\%20English.pdf. Accessed 8 Mar 2021

19. James D (2015) Nonoperative management of adult femoral shaft fracture using the principle of controlled collapse in mission hospital in Central India. CHRISMED J Health Res 2:379-382

20. James D (2016) Challenges in managing ipsilateral femoral neck and shaft fracture at Mission Hospital. CHRISMED J Health Res 3:214-217

21. Meara JG, Leather AJM, Hagander L et al (2015) Global surgery 2030: evidence and solutions for achieving health, welfare, and economic development. Lancet 386:569-624
22. Raykar N, Mukhopadhyay S, Saluja S et al (2019) Implementation of the lancet commission on global surgery in india. Healthc (Amst) 7:4-6

23. Orser BA, Wilson CR, Rotstein AJ et al (2019) Improving access to safe anesthetic care in rural and remote communities in affluent countries. Anesth Analg 129:294-300

24. Enright A, Mitchell R (2019) "Go to the people. live among them". Reflections on anesthetic and surgical care in rural and remote regions. Anesth Analg 129:13-15

25. Panagariya A (2014) The challenges and innovative solutions to rural health dilemma. Ann Neurosci 21:125-127

26. Tongaonkar RR (2003) Scope and limitations of rural surgery. Indian J Surg 65:24-29

27. Surgical care for low-income, rural populations: an alternative delivery model from jan swasthya sahyog, India. http://www. globalsurgery.info/wp-content/uploads/2015/08/India-Case-.pdf. Accessed 1 May 2021

28. Gokavi S. Medical missions in India today. In: http://www. apcwo.org/newsite/resources/missions/MedicalMissionsInIndia Today-DrSunilGokavi-EHA.pdf. Accessed 1 May 2021

29. Badejoko OO, Ijarotimi AO, Osinubi RA et al (2013) Where there is no anaesthetist: the role of obstetrician administered spinal anaesthesia for emergency caesarean section. Trop J Obstet Gynaecol. https://doi.org/10.4314/tjog.v30i1

30. Lewis SR, Nicholson A, Smith AF et al (2014) Physician anaesthetists versus non-physician providers of anaesthesia for surgical patients. Cochrane Database Syst Rev. https://doi.org/10. 1002/14651858.CD010357.pub2

31. Kessler J, Marhofer P, Hopkins PM et al (2015) Peripheral regional anaesthesia and outcome: lessons learned from the last 10 years. Br J Anaesth 114:728-745

32. Cozowicz C, Poeran J, Memtsoudis SG (2015) Epidemiology, trends, and disparities in regional anaesthesia for orthopaedic surgery. Br J Anaesth 115(2):57-67

33. Picard J, Meek T (2010) Complications of regional anaesthesia. Anaesthesia 65(Suppl 1):105-115

34. Adesunkanmi AR (1997) Where there is no anaesthetist: a study of 282 consecutive patients using intravenous, spinal and local infiltration anaesthetic techniques. Trop Doct 27:79-82

35. Harris D (1999) Where there is no anaesthetist. Trop Doct 29:56-56

36. Lokossou T, Zoumenou E, Secka G et al (2007) Anesthesia in French-speaking Sub-Saharan Africa: an overview. Acta Anaesthesiol Belg 58:197-209

37. Enright A (2013) Review article: Safety aspects of anesthesia in under-resourced locations. Can J Anesth 60:152-158

38. Khan FA, Merry AF (2018) Improving anesthesia safety in lowresource settings. Anesth Analg 126:1312-1320

39. Munhall RJ, Sukhani R, Winnie AP (1988) Incidence and etiology of failed spinal anesthetics in a university hospital. Anesth Analg 67:843-848

40. Fettes PDW, Jansson J-R, Wildsmith JAW (2009) Failed spinal anaesthesia: mechanisms, management, and prevention. $\mathrm{Br} \mathrm{J}$ Anaesth 102:739-748

41. Carpenter RL, Caplan RA, Brown DL et al (1992) Incidence and risk factors for side effects of spinal anesthesia. Anesthesiology 76:906-916

Publisher's Note Springer Nature remains neutral with regard to jurisdictional claims in published maps and institutional affiliations. 